\title{
Stochastic Multi-objective Optimisation of Exoskeleton Structures
}

\author{
Anna Reggio ${ }^{1}$ (D) Rita Greco ${ }^{2}$. Giuseppe Carlo Marano ${ }^{1}$. \\ Giuseppe Andrea Ferro ${ }^{1}$
}

Received: 31 March 2020 / Accepted: 29 October 2020 / Published online: 18 November 2020

(c) The Author(s) 2020

\begin{abstract}
In this study, a structural optimisation problem, addressed through a stochastic multiobjective approach, is formulated and solved. The problem deals with the optimal design of exoskeleton structures, conceived as vibration control systems under seismic loading. The exoskeleton structure is assumed to be coupled to an existing primary inner structure for seismic retrofit: the aim is to limit the dynamic response of the primary structure to prevent structural damage. A non-stationary filtered Gaussian white noise stochastic process is taken as the seismic input. Design variables pertain to the mechanical properties (stiffness, damping) of the exoskeleton structure. Two concurrent and competing objective functions are introduced, in order to take into account not only safety performance but also economic cost considerations. The resulting trade-off is solved searching the Pareto front by way of a controlled elitist genetic algorithm, derived from the Non-dominated Sorting Genetic Algorithm-II. Sensitivities of Pareto fronts and Pareto optimal sets to different system parameters are finally investigated by way of a numerical application.
\end{abstract}

Keywords Multi-objective optimisation · Genetic algorithm · Structural optimisation · Random vibration · Vibration control

\footnotetext{
Anna Reggio

anna.reggio@polito.it

Rita Greco

rita.greco@poliba.it

Giuseppe Carlo Marano

giuseppe.marano@polito.it

Giuseppe Andrea Ferro

ferro@polito.it

1 Department of Structural, Geotechnical and Building Engineering, Politecnico di Torino, Turin, Italy

2 Department of Civil, Environmental, Territory, Building and Chemical Engineering, Politecnico di Bari, Bari, Italy 


\section{Introduction}

Engineering structures undergo environmental loads, both natural (seismic action, wind pressure, sea waves, etc.) and anthropic (pedestrian-induced excitation, road and railway traffic, etc.) that are dynamic in time and intrinsically stochastic in nature. When the resulting structural vibration could reach levels impairing serviceability and safety performance, it can be significantly reduced by way of vibration control technologies [1-4].

In earthquake-prone regions, to improve the resilience of the built environment [5], vibration control technologies can be successfully applied to the seismic retrofitting of existing structures. To this end, one of the most promising strategies is currently given by exoskeleton structures. We define an exoskeleton structure as a self-supporting structural system set outside and suitably connected to a primary inner structure, the latter being enhanced or protected, in a general sense, by virtue of this connection. Impressive real applications of exoskeleton structures to building refurbishment projects already exist (e.g. Cannon Place, London, 2011 [6]). The potential for seismic retrofitting is currently under investigation $[7,8]$, mainly with reference to steel braced frame or diagrid exoskeleton structures connected to reinforced concrete buildings [911]. Attractive advantages are envisaged: the suitability to an integrated design of the retrofitting intervention, combining structural safety, energy efficiency and environmental sustainability; limited interference with existing structural and nonstructural components; minimal service/business downtime, as the retrofitting intervention is operated from the outside; consequent limited cost.

In this study, we conceive the exoskeleton structure as a vibration control system under seismic excitation, a "sacrificial appendage" aimed at absorbing seismic loads, reducing the dynamic response of the primary structure and preventing its damage. The intra-connection between primary structure and exoskeleton structure is assumed to be non-dissipative and sufficiently rigid under horizontal loads, e.g. as in the case of frame structures connected at each floor level by means of horizontal steel trusses [10]. The assumption of a rigid coupling essentially characterises the principle of operation in terms of vibration control. Based on such a kinematic constraint, the total force transmitted from the moving base to the mass of the coupled system is split among the primary structure and the exoskeleton structure. If the mechanical properties of the exoskeleton structure are purposely selected, the individual force quota transmitted to the primary structure is greatly reduced, achieving a significant control of its displacement response and overall internal state of stress [9,12]. Greater reductions in the internal forces on the primary structure correspond, on the other hand, to higher internal forces on the exoskeleton structure.

The search for a dynamic coupling is also characteristic of a classical vibration control technology, the Tuned Mass Damper (TMD). In building applications, a TMD is implemented as a relatively small auxiliary mass usually attached to the top of the main structure to be protected [13]. The connection in between is deformable and possibly dissipative. The principle of operation of TMD consists in inducing a vibration energy transfer from the main structure to the auxiliary mass, which dissipates the energy away vibrating out of phase with the structural motion. The effectiveness and robustness of TMD in controlling the earthquake-induced response are, however, 
still questioned in literature and engineering practice, unless the mass ratio of the device can be significantly increased [14-16].

As demonstrated in [9], the control effectiveness shown by an exoskeleton structure depends on an appropriate selection of its mechanical properties (mass, stiffness and damping). Nonetheless, specific design methods and optimisation procedures have yet to be developed. In fact, previous works [17-22] on the optimisation of dissipative inter-connections between adjacent structures are not directly applicable nor immediately transferable to exoskeleton structures, due to different design variables and objective functions.

This paper deals with the optimal design of an exoskeleton structure, coupled to an existing primary structure to the purpose of seismic retrofit. The coupled structures are modelled as a linear time-invariant dynamic system, whose governing equations are given in Sect. 2. The system is composed of two linear viscoelastic oscillators, representative of the reduced-order generalised model of the multi-degree-of-freedom structures [23]. The constitutive hypothesis of linear viscoelasticity is deemed consistent with the aim of preventing structural damage. The intra-connection between the two structures is modelled as a non-dissipative, and in particular rigid, coupling between the two oscillators. The stochastic nature of seismic excitation is explicitly taken into account in the input signal, a non-stationary filtered Gaussian white noise process derived from the Clough and Penzien spectrum [24]. Stochastic optimisation [25-27] appears to be the most convenient approach to the optimal design of vibration control technologies, since neglecting the randomness of dynamic loads may result in suboptimal solutions [28].

A multi-objective optimisation problem is then formulated and its numerical implementation is tackled by use of a genetic algorithm (Sect. 3). Safety and technology cost are considered as concurrent objectives, in order to allow the designer to compare and select the solution that better address not only performance but also economical considerations. Two Objective Functions (OFs) are introduced. The first OF, concerning safety, is probabilistic, reliability based [29] and defined in terms of displacements relative to ground (deformations), which are directly correlated with structural damage (deformation-sensitive). The second OF, concerning cost, is deterministic and given as a weighted combination of the unit costs associated with the mechanical properties of the exoskeleton structure. Since the two OFs are shown to conflict under seismic excitation, the trade-off is solved searching their Pareto front by way of a controlled elitist genetic algorithm, derived from the Non-dominated Sorting Genetic Algorithm-II (NSGA-II) [30-32] and implemented in MATLAB [33]. Strictly speaking, a bi-objective optimisation problem is solved; however, the proposed formulation and numerical implementation are suited to be extended to multiple objectives. This consideration is relevant when the exoskeleton structure is designed to meet multiple performance levels at increasing earthquake intensities, according to the principles of Performance-Based Seismic Design [34].

A numerical application of the optimisation methodology is later presented and discussed. Optimisation results are investigated through parametric analyses, to appreciate their sensitivity to variations in system parameters (Sect. 4). In Sect. 5, the main conclusions from the study are drawn as well as suggestions for future research developments. 
Fig. 1 Structural model: coupled primary-secondary oscillator system

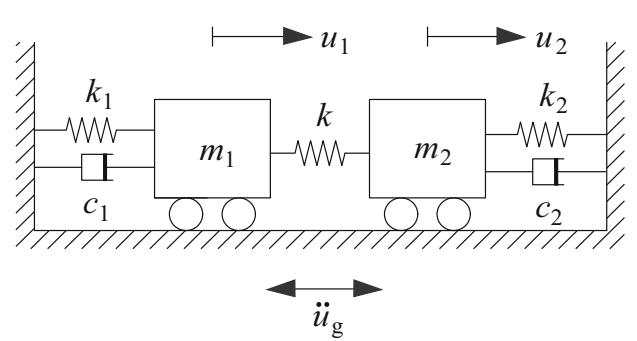

\section{Stochastic Dynamic Analysis}

\subsection{Structural Model}

We consider a single structural system composed of two subsystems, a primary structure and an exoskeleton structure, coupled to each other by way of a non-dissipative intra-connection. Without lack of generality, a suitable mechanical model is given by two single-degree-of-freedom (sdof) oscillators, both having viscoelastic behaviour, coupled via a Hooke spring of stiffness $k$ (Fig. 1).

The dynamic equilibrium of the coupled primary-secondary oscillator system, excited by ground acceleration $\ddot{u}_{\mathrm{g}}(t)$, is drawn in the form

$$
\begin{aligned}
& m_{1} \ddot{u}_{1}+c_{1} \dot{u}_{1}+k_{1} u_{1}=-m_{1} \ddot{u}_{\mathrm{g}}+k\left(u_{2}-u_{1}\right), \\
& m_{2} \ddot{u}_{2}+c_{2} \dot{u}_{2}+k_{2} u_{2}=-m_{2} \ddot{u}_{\mathrm{g}}-k\left(u_{2}-u_{1}\right),
\end{aligned}
$$

where $u_{1}(t)$ and $u_{2}(t)$ are the displacements, relative to ground, of primary and secondary oscillator, respectively, with the overdot denoting differentiation with respect to time $t ; m_{1}, k_{1}$ and $c_{1}$ are the mass, stiffness and damping coefficients of primary oscillator; $m_{2}, k_{2}$ and $c_{2}$ are the mass, stiffness and damping coefficients of secondary oscillator.

The hypothesis of a rigid coupling between primary and secondary oscillator can be viewed as the limit case of the Hooke spring with stiffness coefficient tending to infinity, $k \rightarrow \infty$. It follows $u_{2} \rightarrow u_{1}$ and, to the limit, Eq. (1) are replaced by the dynamic equilibrium equation of an sdof system:

$$
\left(m_{1}+m_{2}\right) \ddot{u}_{1}+\left(c_{1}+c_{2}\right) \dot{u}_{1}+\left(k_{1}+k_{2}\right) u_{1}=-\left(m_{1}+m_{2}\right) \ddot{u}_{\mathrm{g}}
$$

The following parameters are defined:

$$
\omega_{1}=\sqrt{\frac{k_{1}}{m_{1}}}, \quad \zeta_{1}=\frac{c_{1}}{2 \sqrt{k_{1} m_{1}}}, \quad \zeta_{2}=\frac{c_{2}}{2 \sqrt{k_{2} m_{2}}}, \quad \mu=\frac{m_{2}}{m_{1}}, \quad \alpha=\frac{\omega_{2}}{\omega_{1}},
$$

which denote, respectively, the uncoupled natural frequency $\omega_{1}$ and damping ratio $\zeta_{1}$ of the primary oscillator; the uncoupled damping ratio $\zeta_{2}$ of the secondary oscillator; the mass ratio $\mu$ and the frequency ratio $\alpha$ between the two oscillators, being $\omega_{2}=\sqrt{\frac{k_{2}}{m_{2}}}$ 


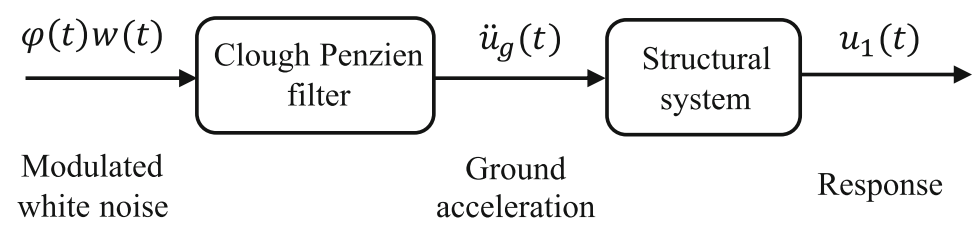

Fig. 2 Block diagram representation of the stochastic seismic excitation model

the uncoupled natural frequency of the secondary oscillator. Equation (2) is rewritten accordingly as:

$$
(1+\mu) \ddot{u}_{1}+\left(2 \zeta_{1} \omega_{1}+2 \zeta_{2} \alpha \omega_{1} \mu\right) \dot{u}_{1}+\left(\omega_{1}^{2}+\alpha^{2} \omega_{1}^{2} \mu\right) u_{1}=-(1+\mu) \ddot{u}_{\mathrm{g}} .
$$

\subsection{Stochastic Excitation Model}

Strong ground motions caused by earthquakes are influenced by a number of random phenomena correlated with the complex and irregular nature of the formations traversed by seismic waves. To take into account such an uncertain nature, it is appropriate to model seismic excitation as a stochastic process.

In this study, the input to the structural system is the ground acceleration $\ddot{u}_{\mathrm{g}}(t)$ due to earthquake. It is modelled as a non-stationary filtered Gaussian stochastic process, as per the Clough and Penzien Power Spectral Density (PSD) function for free-field ground motions [24]:

$$
\begin{aligned}
& \ddot{u}_{\mathrm{g}}=-\omega_{\mathrm{p}}^{2} u_{\mathrm{p}}-2 \zeta_{\mathrm{p}} \omega_{\mathrm{p}} \dot{u}_{\mathrm{p}}+\omega_{\mathrm{f}}^{2} u_{\mathrm{f}}+2 \zeta_{\mathrm{f}} \omega_{\mathrm{f}} \dot{u}_{\mathrm{f}}, \\
& \ddot{u}_{\mathrm{p}}+\omega_{\mathrm{p}}^{2} u_{\mathrm{p}}+2 \zeta_{\mathrm{p}} \omega_{\mathrm{p}} \dot{u}_{\mathrm{p}}=\omega_{\mathrm{f}}^{2} u_{\mathrm{f}}+2 \zeta_{\mathrm{f}} \omega_{\mathrm{f}} \dot{u}_{\mathrm{f}}, \\
& \ddot{u}_{\mathrm{f}}+2 \zeta_{\mathrm{f}} \omega_{\mathrm{f}} \dot{u}_{\mathrm{f}}+\omega_{\mathrm{f}}^{2} u_{\mathrm{f}}=-\phi(t) w(t) .
\end{aligned}
$$

In (5), $w(t)$ is a stationary Gaussian white noise with constant PSD $S_{0}$ and zero mean, uniformly modulated by function $\phi(t) ; u_{\mathrm{f}}(t)$ is the response of a first filter, with frequency $\omega_{\mathrm{f}}$ and damping ratio $\zeta_{\mathrm{f}} ; u_{\mathrm{p}}(t)$ is the response of a second filter, with frequency $\omega_{\mathrm{p}}$ and damping ratio $\zeta_{\mathrm{p}}$. The modulation function $\phi(t)$ is taken as the piece-wise function proposed by Jennings et al. [35-38], whose formula is

$$
\phi(t)= \begin{cases}\left(t / t_{1}\right)^{2}, & t<t_{1} \\ 1, & t_{2} \leq t \leq t_{1} \\ \exp \left(-\theta\left(t-t_{2}\right)\right), & t>t_{2}\end{cases}
$$

being $t_{1}$ and $t_{2}$ the start and end times of the strong shaking phase and $\theta$ the coefficient controlling the shape of the decaying end of the function.

A block diagram representation of the stochastic excitation model is given in Fig. 2. 


\subsection{State-Space Governing Equations and System Response}

By introducing the stochastic excitation (5) into the dynamic equilibrium Eq. (4), the augmented dynamic system reads

$$
\begin{aligned}
& (1+\mu) \ddot{u}_{1}+\left(2 \zeta_{1} \omega_{1}+2 \zeta_{2} \alpha \omega_{1} \mu\right) \dot{u}_{1}+\left(\omega_{1}^{2}+\alpha^{2} \omega_{1}^{2} \mu\right) u_{1}=-(1+\mu) \ddot{u}_{\mathrm{g}}, \\
& \ddot{u}_{\mathrm{g}}=-\omega_{\mathrm{p}}^{2} u_{\mathrm{p}}-2 \zeta_{\mathrm{p}} \omega_{\mathrm{p}} \dot{u}_{\mathrm{p}}+\omega_{\mathrm{f}}^{2} u_{\mathrm{f}}+2 \zeta_{\mathrm{f}} \omega_{\mathrm{f}} \dot{u}_{\mathrm{f}}, \\
& \ddot{u}_{\mathrm{p}}+\omega_{\mathrm{p}}^{2} u_{\mathrm{p}}+2 \zeta_{\mathrm{p}} \omega_{\mathrm{p}} \dot{u}_{\mathrm{p}}=\omega_{\mathrm{f}}^{2} u_{\mathrm{f}}+2 \zeta_{\mathrm{f}} \omega_{\mathrm{f}} \dot{u}_{\mathrm{f}} \\
& \ddot{u}_{\mathrm{f}}+2 \zeta_{\mathrm{f}} \omega_{\mathrm{f}} \dot{u}_{\mathrm{f}}+\omega_{\mathrm{f}}^{2} u_{\mathrm{f}}=-\phi(t) w(t)
\end{aligned}
$$

It is suited to state-space representation, given in the form:

$$
\begin{aligned}
& \dot{\mathbf{z}}=\mathbf{A z}+\mathbf{B} e(t), \\
& \mathbf{y}=\mathbf{C z} .
\end{aligned}
$$

In the state equation $(8 \mathrm{a}), \mathbf{z}(t)=\left[u_{1}(t), u_{p}(t), u_{f}(t), \dot{u}_{1}(t), \dot{u}_{p}(t), \dot{u}_{f}(t)\right]^{\mathrm{T}}$ is the state vector;

$$
\mathbf{A}=\left[\begin{array}{cccccc}
0 & 0 & 0 & 1 & 0 & 0 \\
0 & 0 & 0 & 0 & 1 & 0 \\
0 & 0 & 0 & 0 & 0 & 1 \\
-\frac{\left(\omega_{1}^{2}+\alpha^{2} \omega_{1}^{2} \mu\right)}{1+\mu} & \omega_{\mathrm{p}}^{2} & -\omega_{\mathrm{f}}^{2}-\frac{\left(2 \zeta_{1} \omega_{1}+2 \zeta_{2} \alpha \omega_{1} \mu\right)}{1+\mu} & 2 \zeta_{\mathrm{p}} \omega_{\mathrm{p}} & -2 \zeta_{\mathrm{f}} \omega_{\mathrm{f}} \\
0 & -\omega_{\mathrm{p}}^{2} & \omega_{\mathrm{f}}^{2} & 0 & -2 \zeta_{\mathrm{p}} \omega_{\mathrm{p}} & 2 \zeta_{\mathrm{f}} \omega_{\mathrm{f}} \\
0 & 0 & -\omega_{\mathrm{f}}^{2} & 0 & 0 & -2 \zeta_{\mathrm{f}} \omega_{\mathrm{f}}
\end{array}\right]
$$

is the state matrix, function of the system parameters; $\mathbf{B}=\left[\begin{array}{ll}0,0,0,0,0,-1 & 0, \mathrm{~T}\end{array}\right.$ is the input matrix; and $e(t)=\phi(t) w(t)$ is the applied input process. In the output equation $(8 \mathrm{~b}), \mathbf{y}(t)=\left[u_{1}(t), \dot{u}_{1}(t), \ddot{u}_{1}^{\mathrm{a}}(t)\right]^{T}$ is the output vector, listing structural displacement $u_{1}(t)$ and velocity $\dot{u}_{1}(t)$, both relative to ground, and structural absolute acceleration $\ddot{u}_{1}^{\mathrm{a}}(t)=\ddot{u}_{1}(t)+\ddot{u}_{\mathrm{g}}(t)$;

$$
\mathbf{C}=\left[\begin{array}{cccccc}
1 & 0 & 0 & 0 & 0 & 0 \\
0 & 0 & 0 & 1 & 0 & 0 \\
-\frac{\left(\omega_{1}^{2}+\alpha^{2} \omega_{1}^{2} \mu\right)}{1+\mu} & 0 & 0 & -\frac{\left(2 \zeta_{1} \omega_{1}+2 \zeta_{2} \alpha \omega_{1} \mu\right)}{1+\mu} & 0 & 0
\end{array}\right]
$$

is the output matrix.

Given the assumptions of zero-mean, uniformly modulated, white noise input process and zero initial conditions, the system response $\mathbf{z}(t)$ is, in turn, a zero-mean non-stationary stochastic vector process, whose complete description can be given in terms of time-variant covariance matrix $\mathbf{R}_{\mathbf{z z}}(t)$. The latter reduces to $\mathbf{R}_{\mathbf{z z}}=\mathrm{E}(\mathbf{z z})$, the symbol $\mathrm{E}(\cdot)$ denoting the expected value operator, and can be evaluated numerically 
[39] by solving the matrix differential equation [40,41]:

$$
\dot{\mathbf{R}}_{\mathbf{Z Z}}=\mathbf{A} \mathbf{R}_{\mathbf{Z Z}}+\mathbf{R}_{\mathbf{Z Z}} \mathbf{A}^{\mathrm{T}}+2 \pi S_{0} \phi^{2}(t) \mathbf{B B}^{\mathrm{T}} .
$$

Once the covariance matrix $\mathbf{R}_{\mathbf{z z}}(t)$ of the state vector is known, the covariance matrix $\mathbf{R}_{\mathbf{y y}}(t)$ of the output vector is subsequently obtained as

$$
\mathbf{R}_{\mathbf{y y}}=\mathrm{E}\left(\mathbf{y} \mathbf{y}^{\mathrm{T}}\right)=\mathbf{C E}\left(\mathbf{z z}^{\mathrm{T}}\right) \mathbf{C}^{\mathrm{T}}
$$

\section{Multi-objective Optimisation Problem}

In this Section, the optimal design of the exoskeleton structure is tackled as a multiobjective optimisation problem. The mathematical formulation of the problem and its numerical implementation in a genetic algorithm are discussed.

\subsection{Design Variables}

The primary structure is assumed to be known, while the exoskeleton structure is designed through the optimisation problem. Referring to the coupled system introduced in Sect. 2.1, the uncoupled natural frequency $\omega_{1}$ and damping ratio $\zeta_{1}$ of the primary oscillator are considered as given data; mass ratio $\mu$ is taken as fixed; frequency ratio $\alpha$ and the uncoupled damping ratio $\zeta_{2}$ of the secondary oscillator play the role of design variables. Design variables are listed in the design vector $\mathbf{b}=\left(\alpha, \zeta_{2}\right)^{\mathrm{T}}$.

\subsection{Objective Functions}

The optimal design of the exoskeleton structure is aimed at the minimisation of two quantities of interest: the maximum displacement relative to ground of the primary structure and the cost of the exoskeleton structure.

Concerning the first objective, we note that the displacement relative to ground represents the engineering demand parameter which the damage of the primary structure is correlated with. For the displacement response process $u_{1}(t)$, we assume the existence of a safe domain limited between an upper bound level $+u_{1 \max }$ and a lower bound level $-u_{1 \max }$, i.e. a symmetric double-barrier domain $\pm u_{1 \max }$ with threshold $u_{1 \max }>0$. Structural failure clearly occurs when $u_{1}(t)$ exceeds for the first time either the upper or the lower bound level (event of first-passage failure). Adopting a reliability-based optimisation approach, the first OF is defined as the maximum absolute displacement $u_{1 \max }$ that is exceeded with a probability of failure not greater than a prescribed value $\bar{P}_{\mathrm{f}}$ in a fixed time interval $T$ :

$$
O F_{1}:=u_{1 \max }: P_{\mathrm{f}}\left(u_{1 \max }, T\right)=\mathcal{P}\left\{\left|u_{1}(t)\right|>u_{1 \max } ; 0 \leq t \leq T\right\} \leq \bar{P}_{\mathrm{f}} .
$$

The complement to one of the probability of failure $P_{\mathrm{s}}=1-P_{\mathrm{f}}$ is the probability of survival, or reliability, of the primary structure. 
There are no exact analytical solutions yet for the probability of failure defined in (13), although approximate solutions are available [40,41]. For wide-band processes and/or high threshold levels, it is reasonable to assume that the events of threshold upcrossing are rare and independent: this makes the process counting the number of upcrossings a Poisson Process (Poisson approximation of the first-passage problem [41]). Starting from zero initial conditions, the probability of survival is the probability of no threshold upcrossing in time interval $(0, T]$, hence the probability of failure can be written as:

$$
P_{\mathrm{f}}\left(u_{1 \max }, T\right)=1-P_{\mathrm{s}}\left(u_{1 \max }, T\right)=1-\exp \left(-\int_{0}^{T} v\left(u_{1 \max }, t\right) \mathrm{d} t\right)
$$

in which $v\left(u_{1 \max }, t\right)=2 v^{+}\left(u_{1 \max }, t\right)$ is the unconditional upcrossing rate of a symmetric double-barrier problem, equal to twice the upcrossing rate $v^{+}\left(u_{1 \max }, t\right)$ of the single-barrier problem. For a non-stationary zero-mean Gaussian process, it holds [41]:

$$
\begin{gathered}
v^{+}\left(u_{1 \max }, t\right)=\frac{\sigma_{\dot{u}_{1}}(t)}{\sigma_{u_{1}}(t)} \exp \left(\frac{-u_{1 \max }^{2}}{2 \sigma_{u_{1}}^{2}(t)}\right) \\
{\left[\frac{\rho_{u_{1} \dot{u}_{1}}(t, t) u_{1 \max }}{\sqrt{2 \pi} \sigma_{u_{1}}(t)} \Phi\left(\frac{\rho_{u_{1} \dot{u}_{1}}(t, t) u_{1 \max }}{\sqrt{1-\rho_{u_{1} \dot{u}_{1}}^{2}(t, t)} \sigma_{u_{1}}(t)}\right)\right.} \\
\left.+\frac{\sqrt{1-\rho_{u_{1} \dot{u}_{1}}^{2}(t, t)}}{2 \pi} \exp \left(\frac{-\rho_{u_{1} \dot{u}_{1}}^{2}(t, t) u_{1 \max }^{2}}{2\left(1-\rho_{u_{1} \dot{u}_{1}}^{2}(t, t)\right) \sigma_{u_{1}}^{2}(t)}\right)\right],
\end{gathered}
$$

where $\sigma_{u_{1}}$ and $\sigma_{\dot{u}_{1}}$ are the standard deviations of $u_{1}(t)$ and $\dot{u}_{1}(t)$, respectively; $\rho_{u_{1} \dot{u}_{1}}(t, t)$ is the correlation coefficient; $\Phi(\cdot)$ is the cumulative Gaussian distribution function. The stochastic constraint condition in Eq. (13) is finally expressed as:

$$
g\left(u_{1 \max }, T\right)=\int_{0}^{T} v\left(u_{1 \max }, t\right) \mathrm{d} t+\log \left(1-\bar{P}_{\mathrm{f}}\right) \leq 0
$$

Concerning the second objective, a sound and representative cost function is sought for the exoskeleton structure, in order to compare the economic investment required by competing design strategies. To this purpose, the cost components considered as meaningful in this study are associated with the mechanical properties of the secondary oscillator, i.e. coefficients $k_{2}$ and $c_{2}$. Coefficient $k_{2}$ represents the generalised stiffness shown by the exoskeleton structure when subjected to the lateral forces due to earthquake. It depends on the material and geometric properties of structural members and is correlated with design and sizing. Coefficient $c_{2}$ represents the generalised damping of the exoskeleton structure. It characterises the energy dissipation properties resulting from both structural members (structural damping) and possible ad hoc devices, or dampers (supplemental damping). 
A cost function is defined as a linear combination of stiffness and damping coefficients $k_{2}$ and $c_{2}$ :

$$
C=C\left(k_{2}, c_{2}\right)=k_{2}+\lambda c_{2}
$$

where $\lambda$ is a cost parameter, defined as the ratio between the unit costs associated, respectively, with $c_{2}$ and $k_{2}$ :

$$
\lambda=\frac{\text { unit } \operatorname{cost}\left(c_{2}\right)}{\text { unit } \operatorname{cost}\left(k_{2}\right)} .
$$

Cost parameter $\lambda$ is used to take account of the generally different (higher) unit cost shown by damping components compared to stiffness components. The cost function (17), expressed per unit mass of the primary oscillator and rearranged by introducing the system parameters (3), is taken as the second OF:

$$
O F_{2}:=\frac{C}{m_{1}}=\mu\left(\alpha^{2} \omega_{1}^{2}+2 \zeta_{2} \alpha \omega_{1} \lambda\right) .
$$

\subsection{Problem Formulation and Numerical Implementation}

A dual criteria optimisation problem is formulated by requiring $O F_{1}(13)$ and $O F_{2}$ (19) to be minimised over the domain admissible for the design vector $\mathbf{b}$ :

$$
\begin{aligned}
& \min \mathbf{v}(\mathbf{b})=\left[\begin{array}{l}
O F_{1}(\mathbf{b}) \\
O F_{2}(\mathbf{b})
\end{array}\right], \\
& O F_{1}(\mathbf{b})=u_{1 \max }(\mathbf{b}), \quad O F_{2}(\mathbf{b})=\frac{C(\mathbf{b})}{m_{1}}, \\
& \mathbf{b}=\left[\alpha, \zeta_{2}\right]^{\mathrm{T}}, \\
& \text { subject to } g(\mathbf{b})=\int_{0}^{T} v\left(u_{1 \max }(\mathbf{b}), t\right) d t+\log \left(1-\bar{P}_{\mathrm{f}}\right) \leq 0 \text {, } \\
& \alpha^{\mathrm{L}} \leq \alpha \leq \alpha^{\mathrm{U}} \text { with } \alpha^{\mathrm{L}}, \alpha^{\mathrm{U}} \in \mathbb{R}^{+}, \\
& \zeta_{2}^{\mathrm{L}} \leq \zeta_{2} \leq \zeta_{2}^{\mathrm{U}} \text { with } \zeta_{2}^{\mathrm{L}}, \zeta_{2}^{\mathrm{U}} \in \mathbb{R}_{0}^{+} \text {. }
\end{aligned}
$$

Objective functions $O F_{1}(13)$ and $O F_{2}$ (19) are expected to conflict with each other: increments of the design variables $\alpha$ and $\zeta_{2}$ lead to reduce $O F_{1}$ (the maximum displacement of the primary structure) [9] and to increase $O F_{2}$ (the cost of the exoskeleton structure). In such a case of conflicting objectives, a set of alternative optimal solutions can be found in accordance with the concept of Pareto optimality [42]. These solutions are said to be non-dominated or non-inferior optimal solutions in the sense that, for each of them, one OF cannot be improved unless degrading another one. Generally speaking, given two candidate design vectors $\mathbf{b}_{k}$ and $\mathbf{b}_{l}$ and defined 
the two corresponding objective function vectors

$$
\begin{aligned}
\mathbf{v}\left(\mathbf{b}_{k}\right) & =\left[O F_{1}\left(\mathbf{b}_{k}\right), \ldots, O F_{m}\left(\mathbf{b}_{k}\right)\right]^{\mathrm{T}}, \\
\mathbf{v}\left(\mathbf{b}_{l}\right) & =\left[O F_{1}\left(\mathbf{b}_{l}\right), \ldots, O F_{m}\left(\mathbf{b}_{l}\right)\right]^{\mathrm{T}},
\end{aligned}
$$

vector $\mathbf{v}\left(\mathbf{b}_{k}\right)$ is said to dominate vector $\mathbf{v}\left(\mathbf{b}_{l}\right)$ (denoted as $\mathbf{v}\left(\mathbf{b}_{k}\right) \prec \mathbf{v}\left(\mathbf{b}_{l}\right)$ ) if

$$
\begin{aligned}
& O F_{i}\left(\mathbf{b}_{k}\right) \leq O F_{i}\left(\mathbf{b}_{l}\right) \quad \forall i \in[1, \ldots, m] \\
& \wedge \exists j \in[1, \ldots, m]: O F_{j}\left(\mathbf{b}_{k}\right)<O F_{j}\left(\mathbf{b}_{l}\right) .
\end{aligned}
$$

If no feasible solution $\mathbf{v}\left(\mathbf{b}_{k}\right)$ does exist that dominates solution $\mathbf{v}\left(\mathbf{b}_{l}\right)$, then $\mathbf{v}\left(\mathbf{b}_{l}\right)$ is classified as a non-dominated or Pareto optimal solution. The collection of all the Pareto optimal solutions in the space of design variables is known as Pareto optimal set; in the space of OFs, the locus corresponding to the Pareto optimal set is called Pareto front. Each point on the front represents a trade-off between multiple objectives and, if no preference information is given, no trade-off can be valued better that the others. Only the human decision maker (i.e. the structural designer in the present case) can solve this uncertainty by selecting the single solution on the front that better agrees with his/her own subjective preferences.

The Pareto front for the bi-objective optimisation problem (20) is found by using the controlled elitist genetic algorithm implemented in MATLAB function gamultiobj [33], which is a variant of the Non-dominated Sorting Genetic Algorithm-II (NSGAII) [30-32]. Genetic algorithms have been long recognised as robust, reliable and computationally efficient tools for multi-objective structural optimisation [43-45]. Compared to a typical elitist genetic algorithm, the controlled elitist genetic algorithm adopted in the present study not always favours individuals with better fitness values, but also includes individuals that help increase the diversity of the population, even if they have a lower fitness value. This process maintains the diversity of population, which improves crucially the convergence to an optimal Pareto front.

\section{Results and Discussion}

In this section, a numerical application of the methodology formulated in Section 3 is shown and discussed. The design parameters for the optimisation problem are given below.

Concerning the primary oscillator, fixed parameters are the uncoupled natural frequency $\omega_{1}=10.47 \mathrm{rad} / \mathrm{s}$ (corresponding period $T_{1}=0.6 \mathrm{~s}$ ) and damping ratio $\zeta_{1}=0.05$. The mass ratio between the secondary oscillator and the primary oscillator is taken as $\mu=0.001$. The design vector $\mathbf{b}$, listing frequency ratio $\alpha$ and the damping ratio $\zeta_{2}$ of the secondary oscillator, is assumed to vary in the admissible domain $\Omega_{\mathbf{b}}=\left\{\mathbf{b}=\left[\alpha, \zeta_{2}\right]^{\mathrm{T}}: \alpha \in\left[10^{-1}, 10^{2}\right] \wedge \zeta_{2} \in[0,1]\right\}$. Since $\zeta_{2}$ represents the generalised damping ratio of the exoskeleton structure in the reduced-order dynamic model, its variation range is chosen to encompass also the case in which the exoskeleton struc- 
Table 1 Parameters of the stochastic seismic excitation model

Table 2 Setup parameters used for the controlled elitist genetic algorithm implemented in MATLAB function gamultiobj

\begin{tabular}{lll}
\hline$\omega_{\mathrm{f}}$ & 20 & $\mathrm{rad} / \mathrm{s}$ \\
$\zeta_{\mathrm{f}}$ & 0.7 & \\
$\omega_{\mathrm{p}}$ & 3 & $\mathrm{rad} / \mathrm{s}$ \\
$\zeta_{\mathrm{p}}$ & 0.3 & \\
$S_{0}$ & 294.30 & $\mathrm{~cm}^{2} / \mathrm{s}^{3}$ \\
$t_{1}$ & 2 & $\mathrm{~s}$ \\
$t_{2}$ & 12 & $\mathrm{~s}$ \\
$\theta$ & 0.4 & $\mathrm{~s}^{-1}$ \\
\hline
\end{tabular}

Maximum number of generations

400

Population size

50

Crossover fraction

0.8

Pareto fraction

ture is provided with energy dissipation devices. Values of $\zeta_{2}$ up to 1.0 are able to model such a supplemental damping in addition to the mere structural damping.

Regarding seismic excitation, parameters of the stochastic model are indicated in Table 1.

As to the MATLAB function gamultiobj, setup parameters are listed in Table 2. In the stochastic constraint condition (16), the considered probability of failure $\bar{P}_{\mathrm{f}}$ is equal to $10^{-3}$ in $T=100 \mathrm{~s}$, being such a value of $T$ conservative for seismic excitation. In $O F_{2}(19)$, the cost parameter is $\lambda=10 \frac{\mathrm{N} / \mathrm{m}}{\mathrm{Ns} / \mathrm{m}}$.

Surface plots in Fig. 3a, b depict $O F_{1}$ and $O F_{2}$ versus the design variables $\alpha$ and $\zeta_{2}$ spanning the admissible domain $\Omega_{\mathbf{b}}$. By inspecting the surfaces, it becomes apparent that $O F_{1}$ and $O F_{2}$ have antithetical trends: as $O F_{1}$, i.e. the maximum displacement response of the primary oscillator, decreases, $O F_{2}$, i.e. the cost associated with the secondary oscillator, increases. A trade-off problem is hence posed between improving the performance of the controlled primary structure, minimising its displacement response, and limiting the cost of the exoskeleton structure.

Contour plots matching the surface plots of Fig. 3a, b are reported in Fig. 4a, b, respectively, to appreciate the sensitivity of $O F_{1}$ and $O F_{2}$ to variations of $\alpha$ and $\zeta_{2}$. From Fig. 4a, the response function $O F_{1}$ appears to be reduced by increments of $\alpha$, particularly pronounced when $\alpha>10$, and by increments of $\zeta_{2}$. Conversely, the same increments cause the cost function $O F_{2}$ to increase steeply in Fig. 4b. Both $O F_{1}$ and $O F_{2}$ are, broadly speaking, more sensitive to variations of $\alpha$ than of $\zeta_{2}$. Given a fixed value of the mass ratio between the two oscillators, increments and decrements of frequency ratio $\alpha$ correspond to increments and decrements of the stiffness of the secondary oscillator.

In Fig. 4c, $O F_{1}$ is normalised with respect to the maximum displacement of the so-called uncontrolled primary oscillator, i.e, the primary oscillator considered as uncoupled from the secondary oscillator. Values smaller than one imply a reduction in the primary oscillator response by virtue of the coupling to the secondary oscilla- 
(a)

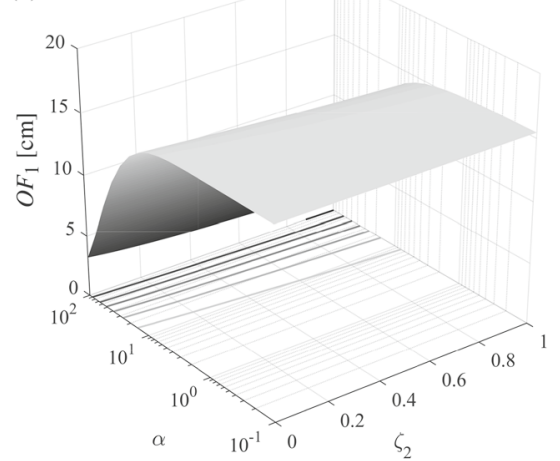

(b)

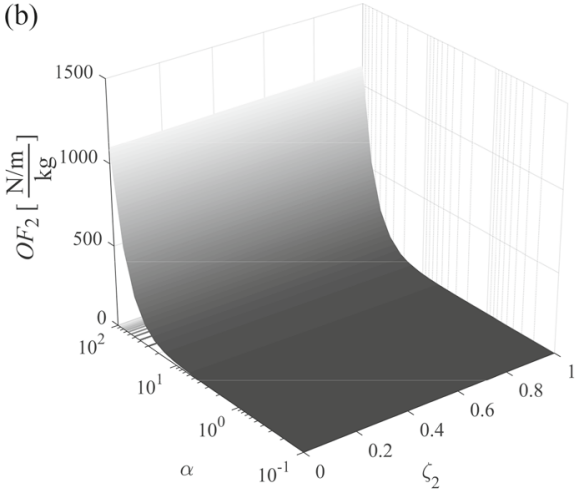

Fig. 3 Surface plots of $O F_{1}$ (a) and $O F_{2}(\mathbf{b})$ in the space of design variables $\alpha$ and $\zeta_{2}$. It is assumed: $\omega_{1}=10.47 \mathrm{rad} / \mathrm{s}\left(T_{1}=0.6 \mathrm{~s}\right), \zeta_{1}=0.05, \mu=0.001$

(a)

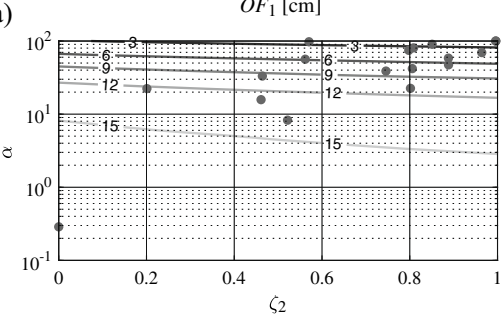

(c)

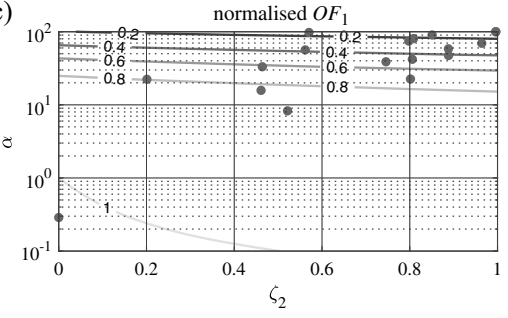

(d)

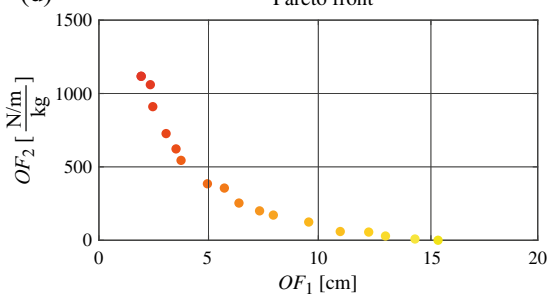

(b)

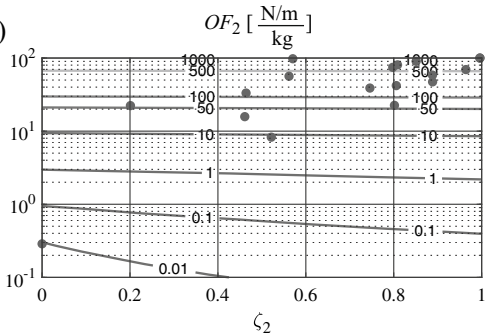

Fig. 4 Contour plots of $O F_{1}(\mathbf{a}), O F_{2}$ (b) and normalised $O F_{1}(\mathbf{c})$ in the space of design variables $\alpha$ and $\zeta_{2}$; Pareto front (d) in the space of OFs and Pareto optimal solutions (e) in the space of design variables $\alpha$ and $\zeta_{2}$. It is assumed: $\omega_{1}=10.47 \mathrm{rad} / \mathrm{s}\left(T_{1}=0.6 \mathrm{~s}\right), \zeta_{1}=0.05, \mu=0.001$

(e)

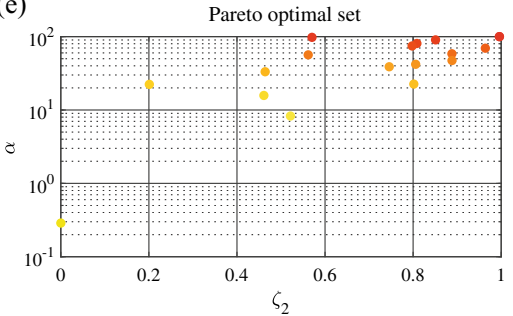


Table 3 Pareto front, values of $O F_{1}$ and $O F_{2}$ and corresponding optimal design variables. It is assumed: $\omega_{1}=10.47 \mathrm{rad} / \mathrm{s}\left(T_{1}=0.6 \mathrm{~s}\right)$, $\zeta_{1}=0.05, \mu=0.001$

\begin{tabular}{lllll}
\hline Point & $O F_{1}(\mathrm{~cm})$ & $O F_{2}\left(\frac{\mathrm{N} / \mathrm{m}}{\mathrm{kg}}\right)$ & $\alpha(-)$ & $\zeta_{2}(-)$ \\
\hline 1 & 15.46 & 0.01 & 0.29 & 0.00 \\
2 & 14.41 & 8.41 & 8.27 & 0.52 \\
3 & 13.06 & 28.84 & 15.78 & 0.46 \\
4 & 12.30 & 55.71 & 22.35 & 0.20 \\
5 & 11.00 & 59.52 & 22.54 & 0.80 \\
6 & 9.56 & 124.11 & 33.20 & 0.46 \\
7 & 7.95 & 171.52 & 38.84 & 0.75 \\
8 & 7.32 & 200.22 & 41.97 & 0.81 \\
9 & 6.38 & 253.45 & 47.23 & 0.89 \\
10 & 5.72 & 355.59 & 56.41 & 0.56 \\
11 & 4.95 & 384.85 & 58.40 & 0.89 \\
12 & 3.74 & 544.38 & 69.54 & 0.96 \\
13 & 3.51 & 622.44 & 74.58 & 0.80 \\
14 & 3.06 & 726.85 & 80.64 & 0.81 \\
15 & 2.46 & 910.30 & 90.30 & 0.85 \\
16 & 2.34 & 1060.16 & 97.78 & 0.57 \\
17 & 1.93 & 1117.45 & 100.00 & 1.00 \\
\hline
\end{tabular}

tor. From the designer point of view, they prove the effectiveness of the exoskeleton structure in reducing the dynamic response and damage level expected for the primary structure under earthquake loading.

The Pareto front is shown in Fig. 4d, while the Pareto optimal solutions are illustrated in Fig. 4e, with the colour map highlighting the correspondence between the two scatter plots. Besides, the optimal solutions are also overlapped with the contour plots of OFs in Fig. $4 \mathrm{a}-\mathrm{c}$, to observe clearly the relative position. For each point of the Pareto front, values of $O F_{1}, O F_{2}$ and of the optimal design variables are reported in Table 3. Along the front, the response function $O F_{1}$ decreases as the cost function $O F_{2}$ increases, although the rate of variation is not constant: in a first section of the front, the cost increase is less than proportional than the response decrease $\left(\mathrm{OF}_{2}\right.$ increasing from 0.01 to $253.45 \frac{\mathrm{N} / \mathrm{m}}{\mathrm{kg}}$ versus $O F_{1}$ decreasing from 15.46 to $6.38 \mathrm{~cm}$ ); in a second section of the front, the cost increase is more than proportional than the response decrease ( $O F_{2}$ increasing from 253.45 to $1117.45 \frac{\mathrm{N} / \mathrm{m}}{\mathrm{kg}}$ versus $O F_{1}$ decreasing from 6.38 to $1.93 \mathrm{~cm}$ ). From Table 3 , it is observed that the reductions in $O F_{1}$ are mainly due to increasing values of frequency ratio $\alpha$ and, secondarily, to higher values of damping ratio $\zeta_{2}$.

The trade-off between the performance objective $O F_{1}$ and the cost objective $O F_{2}$ is instrumental in achieving the purpose of seismic retrofit. Along the Pareto front, the designer is able to quantify and compare the costs required to achieve progressively better performances of the retrofitted primary structure. Then, the designer is able to select the single Pareto solution that ensures a performance considered as satisfactory at a cost deemed as affordable. The selected Pareto solution identifies the values of 

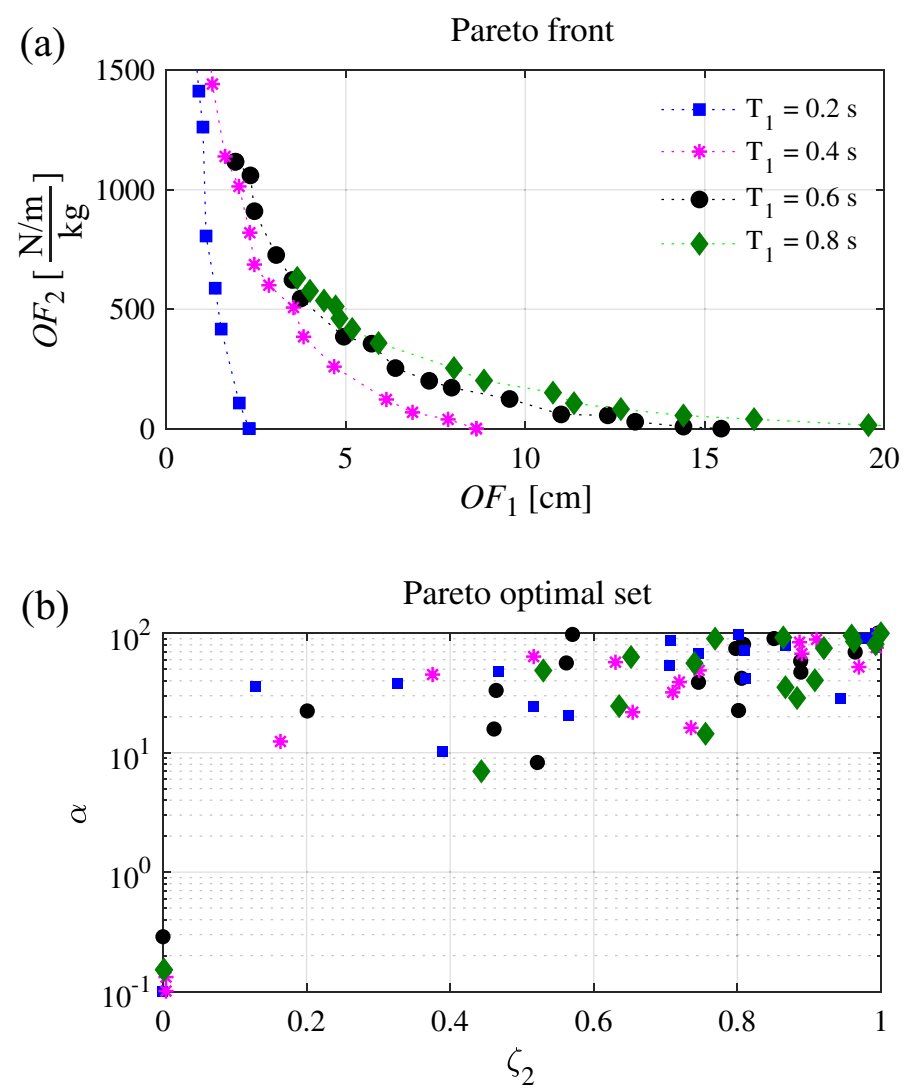

Fig. 5 Sensitivity analysis to variations of the primary oscillator natural period $T_{1}$ : Pareto fronts (a) and Pareto optimal solutions (b). It is assumed: $\mu=0.001$ and $\zeta_{1}=0.05$

frequency ratio $\alpha$ and damping ratio $\zeta_{2}$ that have to be implemented in the design of the exoskeleton structure, i.e. its stiffness and energy dissipation properties, respectively.

Parametric analyses have been carried out to gain more insight into the sensitivity of the Pareto optimal solutions to the design parameters. The analyses have been conducted by varying one parameter at a time, while keeping the other ones fixed to the values assumed in Fig. 4. Sensitivities to natural period $T_{1}$ (Fig. 5) and damping ratio $\zeta_{1}$ (Fig. 6) of the primary oscillator, to mass ratio $\mu$ (Fig. 7) and to cost factor $\lambda$ (Fig. 8) have been investigated.

Figure 5 shows the Pareto fronts and optimal sets obtained for increasing values of the primary oscillator natural period $T_{1}=0.2,0.4,0.6$ and $0.8 \mathrm{~s}$. These values correspond to primary structures with different stiffness properties, from stiff $\left(T_{1}=\right.$ $0.2 \mathrm{~s})$ to relatively flexible $\left(T_{1}=0.8 \mathrm{~s}\right)$. As $T_{1}$ increases, the Pareto fronts in Fig. $5 \mathrm{a}$ move away from the origin of the Cartesian axes. This means that, for a more flexible primary structure, the same response level $\left(O F_{1}\right)$ can be obtained only at the price of a higher cost $\left(\mathrm{OF}_{2}\right)$ of the exoskeleton structure. As apparent from the trend in Fig. $5 \mathrm{~b}$, this higher cost is due to the greater values assumed by the optimal design 


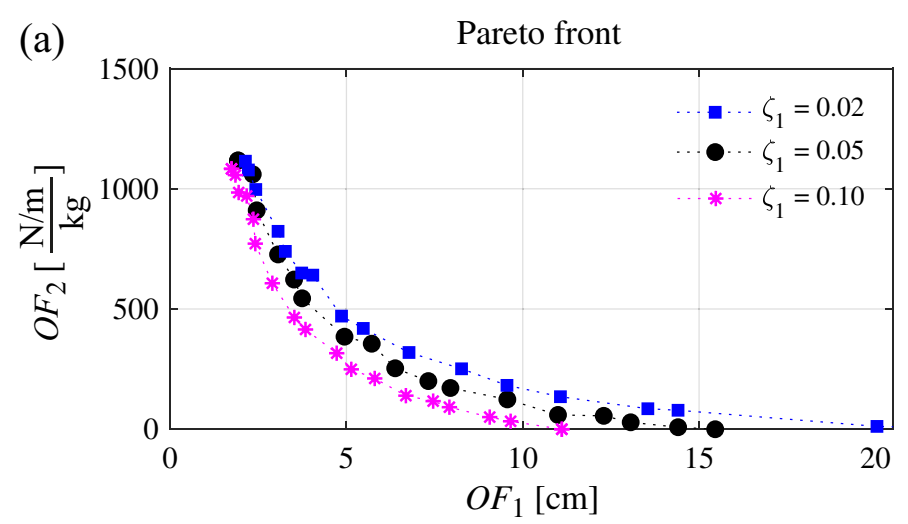

(b)

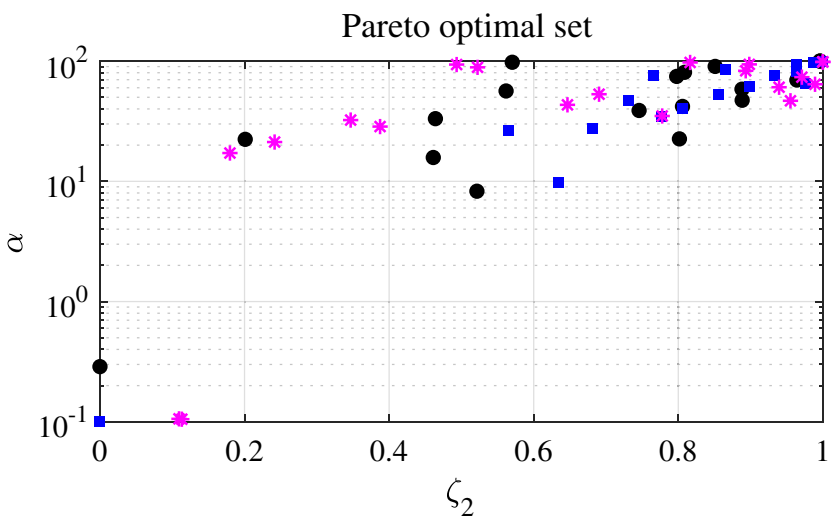

Fig. 6 Sensitivity analysis to variations of the primary oscillator damping ratio $\zeta_{1}$ : Pareto fronts (a) and Pareto optimal solutions (b). It is assumed: $\mu=0.001$ and $\omega_{1}=10.47 \mathrm{rad} / \mathrm{s}\left(T_{1}=0.6 \mathrm{~s}\right)$

variables $\alpha$ and $\zeta_{2}$, which $O F_{2}$ is directly related to, in agreement with Eq. (19). The inspection of the Pareto fronts also shows that their slope decreases with the increasing $T_{1}$, meaning that a greater variation in $O F_{1}$ corresponds to a smaller variation in $O F_{2}$. In particular, by comparing the extreme points of each front: for the stiffest primary structure $\left(T_{1}=0.2 \mathrm{~s}\right)$, the response function $O F_{1}$ is reduced from 2.31 to $0.91 \mathrm{~cm}$, with the cost function $O F_{2}$ rising from 0.00 to $1412.90 \frac{\mathrm{N} / \mathrm{m}}{\mathrm{kg}}$; for the more flexible structure $\left(T_{1}=0.8 \mathrm{~s}\right)$, the response function $O F_{1}$ is reduced from 19.56 to $3.65 \mathrm{~cm}$, with the cost function $O F_{2}$ growing from 14.50 to $630.90 \frac{\mathrm{N} / \mathrm{m}}{\mathrm{kg}}$.

In Fig. 6, the results obtained by varying the primary oscillator damping ratio are presented, with values $\zeta_{1}=0.02,0.05$ and 0.10 assumed in the analyses. As $\zeta_{1}$ increases, the Pareto fronts in Fig. 6a gradually moves toward the origin of the Cartesian axes, meaning that the same response level $\left(O F_{1}\right)$ requires a lower cost $\left(O F_{2}\right)$ of the exoskeleton structure. With regard to the optimal design variables in Fig. 6b, this result corresponds to solutions with smaller damping ratio $\zeta_{2}$. The minimum response level and maximum cost achieved by the optimal solutions are, nevertheless, independent 
(a)

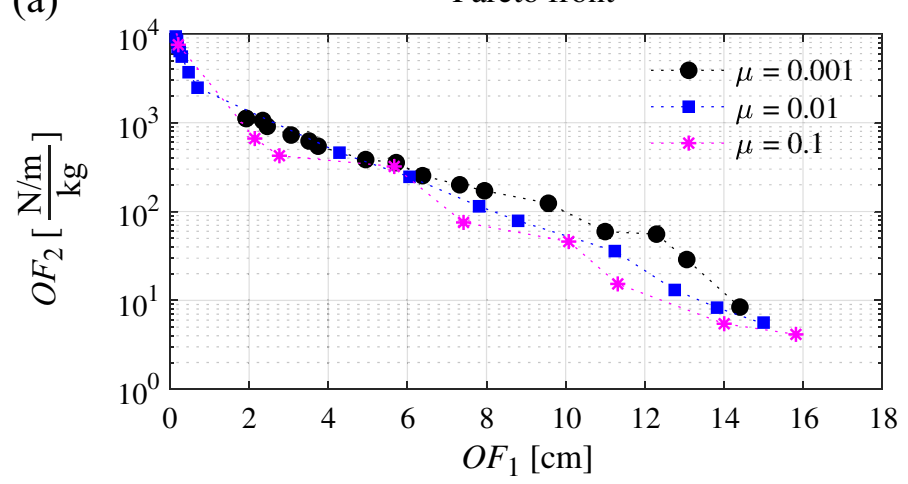

(b)

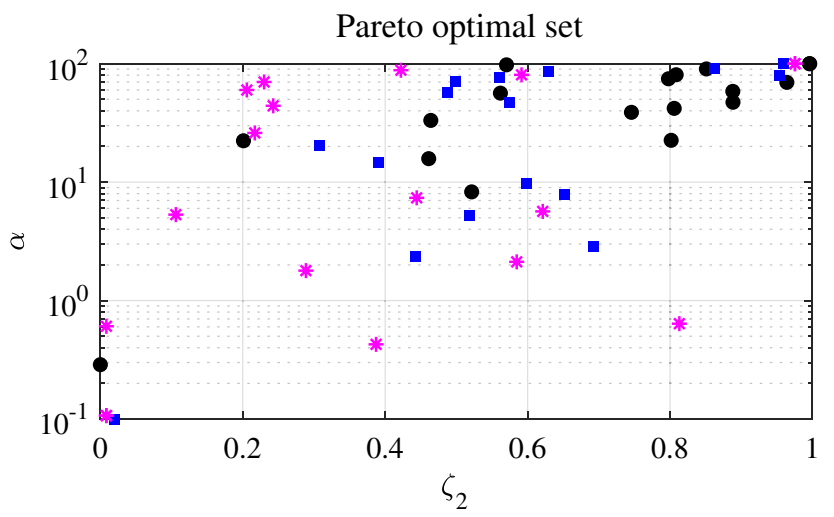

Fig. 7 Sensitivity analysis to variations of mass ratio $\mu$ : Pareto fronts (a) and Pareto optimal solutions (b). It is assumed: $\omega_{1}=10.47 \mathrm{rad} / \mathrm{s}\left(T_{1}=0.6 \mathrm{~s}\right)$ and $\zeta_{1}=0.05$

of $\zeta_{1}$ : the extreme points on the left section of the Pareto fronts are indeed comparable, in terms of both $O F_{1}$ and $O F_{2}$.

Figure 7 illustrates the sensitivity of the optimisation results to variations of the mass ratio $\mu$. The investigated values are $\mu=0.1 \%, 1 \%$ and $10 \%$, which represent progressively more massive exoskeleton structures. By increasing $\mu$, the Pareto fronts in Fig. 7a slightly move toward the origin of the Cartesian axes, meaning that the optimal solutions with a larger mass ratio dominate, in the Pareto sense, the optimal solutions obtained with a smaller mass ratio. Sensitivity to $\mu$ is notable by comparing the left section of the fronts: it can be observed that, the larger the mass ratio $\mu$, the lower the minimum response level (minimum $O F_{1}$ ) achieved by the optimal solutions, although the corresponding cost (maximum $O F_{2}$ ) increases significantly.

Figure 8 shows the effects of different values of the cost parameter, respectively, $\lambda=$ $1,10,100 \frac{\mathrm{N} / \mathrm{m}}{\mathrm{Ns} / \mathrm{m}}$. As $\lambda$ increases, the Pareto fronts in Fig. 8a gradually move away from the origin of the Cartesian axes, meaning that the optimal solutions with a higher cost parameter are dominated, in the Pareto sense, by the optimal solutions with a lower cost parameter. It is worth noting that the minimum value of the response function 


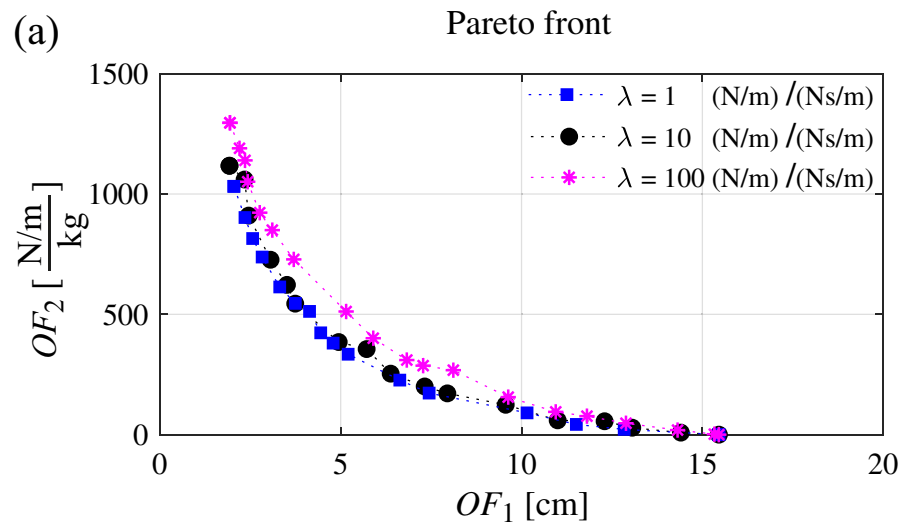

(b)

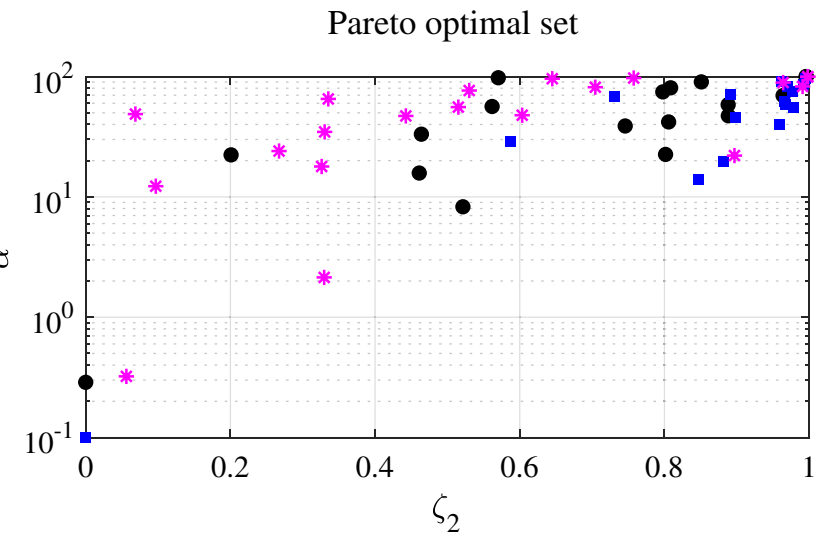

Fig. 8 Sensitivity analysis to variations of cost factor $\lambda$ : Pareto fronts (a) and Pareto optimal solutions (b). It is assumed: $\omega_{1}=10.47 \mathrm{rad} / \mathrm{s}\left(T_{1}=0.6 \mathrm{~s}\right), \zeta_{1}=0.05, \mu=0.001$

$O F_{1}$ achieved by the optimal solutions is basically independent of $\lambda$, whereas the associated value of the cost function $O F_{2}$ grows as $\lambda$ grows.

\section{Conclusions}

In this study, a methodology for the optimal design of linear viscoelastic exoskeleton structures has been developed. The exoskeleton structure has been conceived as a vibration control system, whose mechanical properties (stiffness and damping) are optimised in order to reduce the dynamic response and the expected damage of a primary inner structure under seismic loading.

While the coupled primary and exoskeleton structures are modelled as a deterministic dynamic system, the optimisation problem has been addressed through a stochastic approach, by assuming a non-stationary filtered white noise random process as the seismic input. Two competing OFs have been defined, aiming at trading off the safety 
performance of the primary structure for the economic cost of the exoskeleton structure. Adopting a reliability-based objective function, the safety performance has been expressed in terms of maximum displacement response and associated failure probability. A dual criteria optimisation has been performed by using a controlled elitist genetic algorithm to determine the Pareto front and the corresponding optimal design variables. Although presented with reference to a dual criteria problem, the proposed methodology is suited to be extended to multicriteria optimisation.

The optimisation methodology, first formulated with general scope, has been subsequently applied to a numerical application, in order to investigate features and parameter sensitivity of the obtained optimal solutions. The inspection of the Pareto fronts has proved the effectiveness of the exoskeleton structure in reducing the seismic response and damage level of the primary structure. A trade-off between performance and cost is posed and must be solved by the designer, called to select on the Pareto front the single solution that ensure a performance considered as satisfactory at a cost deemed as acceptable. The selected Pareto solution identifies the values of stiffness and energy dissipation properties that have to be implemented in the design of the exoskeleton structure. Both performance and cost are increased by increments in the stiffness of the exoskeleton structure and, only secondarily, by increments in its energy dissipation properties.

Sensitivity analyses have shown how significantly the system parameters (in particular, primary structure natural period and damping ratio, mass ratio between exoskeleton and primary structure, cost factor) could influence the optimisation results. In detail, dominated optimal solutions (in the Pareto sense) are obtained: with increasing values of the natural period (flexibility) and decreasing values of the damping ratio of the primary structure; by reducing the mass ratio between exoskeleton and primary structure; for higher cost factors.

On the basis of the results obtained, a worthy future research development could be to include uncertainties in structural parameters into the formulated stochastic optimisation methodology. Exoskeleton structures with nonlinear hysteretic behaviour could be of interest as well and would be investigated.

Acknowledgements This research work was partially supported by Dipartimento della Protezione Civile, Presidenza del Consiglio dei Ministri (Italian Civil Protection Department) under project RELUIS-DPC 2019-2021. Anna Reggio is grateful to Politecnico di Torino for funding (Grant 57_RBA19REGANN).

Funding Open access funding provided by Politecnico di Torino within the CRUI-CARE Agreement.

Open Access This article is licensed under a Creative Commons Attribution 4.0 International License, which permits use, sharing, adaptation, distribution and reproduction in any medium or format, as long as you give appropriate credit to the original author(s) and the source, provide a link to the Creative Commons licence, and indicate if changes were made. The images or other third party material in this article are included in the article's Creative Commons licence, unless indicated otherwise in a credit line to the material. If material is not included in the article's Creative Commons licence and your intended use is not permitted by statutory regulation or exceeds the permitted use, you will need to obtain permission directly from the copyright holder. To view a copy of this licence, visit http://creativecommons.org/licenses/by/4.0/. 


\section{References}

1. Housner, G., Bergman, L., Caughey, T., Chassiakos, A., Claus, R., Masri, S.F., Skelton, R.E., Soong, T., Spencer, B.F., Yao, J.: Structural control: past, present, and future. J. Eng. Mech. 123(9), 897-971 (1997). https://doi.org/10.1061/(ASCE)0733-9399(1997)123:9(897)

2. Soong, T., Spencer, B.F.: Supplemental energy dissipation: state-of-the-art and state-of-the-practice. Eng. Struct. 24(3), 243-259 (2002). https://doi.org/10.1016/S0141-0296(01)00092-X

3. Spencer, B.F., Nagarajaiah, S.: State of the art of structural control. J. Struct. Eng. 129(7), 845-856 (2003). https://doi.org/10.1061/(ASCE)0733-9445(2003)129:7(845)

4. Saaed, T.E., Nikolakopoulos, G., Jonasson, J.E., Hedlund, H.: A state-of-the-art review of structural control systems. J. Vib. Control 21(5), 919-937 (2015). https://doi.org/10.1177/1077546313478294

5. Nakashima, M., Lavan, O., Kurata, M., Luo, Y.: Earthquake engineering research needs in light of lessons learned from the 2011 Tohoku earthquake. Earthq. Eng. Eng. Vib. 13(1), 141-149 (2014). https://doi.org/10.1007/s11803-014-0244-y

6. Ove Arup \& Partners Ltd.: Cannon Place Project, London, UK (2011). https://www.arup.com/projects/ cannon-place

7. Caverzan, A., Lamperti Tornaghi, M., Negro, P. (eds.): Proceedings of SAFESUST Workshop-A roadmap for the improvement of earthquake resistance and eco-efficiency of existing buildings and cities. Publications Office of the European Union (2016). https://doi.org/10.2788/499080

8. Rete dei Laboratori Universitari di Ingegneria Sismica (ReLUIS): ReLUIS-DPC 2019-2021 Research Project, funded by the Italian Civil Protection Department. http://www.reluis.it

9. Reggio, A., Restuccia, L., Martelli, L., Ferro, G.A.: Seismic performance of exoskeleton structures. Eng. Struct. 198(article n. 109459) (2019). https://doi.org/10.1016/j.engstruct.2019.109459

10. Formisano, A., Massimilla, A., Di Lorenzo, G., Landolfo, R.: Seismic retrofit of gravity load designed RC buildings using external steel concentric bracing systems. Eng. Fail. Anal. 111(article n. 104485) (2020). https://doi.org/10.1016/j.engfailanal.2020.104485

11. Di Lorenzo, G., Colacurcio, E., Di Filippo, A., Formisano, A., Massimilla, A., Landolfo, R.: State-ofthe-art on steel exoskeletons for seismic retrofit of existing RC buildings. Ingegneria Sismica 37(1), 33-50 (2020)

12. Reggio, A., Restuccia, L., Ferro, G.A.: Feasibility and effectiveness of exoskeleton structures for seismic protection. Procedia Struct. Integrity 9, 303-310 (2018). https://doi.org/10.1016/j.prostr.2018. 06.020

13. Soong, T., Dargush, G.: Passive Energy Dissipation Systems in Structural Engineering. Wiley, Chichester (1997)

14. Rana, R., Soong, T.: Parametric study and simplified design of tuned mass dampers. Eng. Struct. 20(3), 193-204 (1998). https://doi.org/10.1016/S0141-0296(97)00078-3

15. De Angelis, M., Perno, S., Reggio, A.: Dynamic response and optimal design of structures with large mass ratio TMD. Earthq. Eng. Struct. Dyn. 41(1), 41-60 (2012). https://doi.org/10.1002/eqe.1117

16. Reggio, A., Angelis, M.D.: Optimal energy-based seismic design of non-conventional Tuned Mass Damper (TMD) implemented via inter-story isolation. Earthq. Eng. Struct. Dyn. 44, 1623-1642 (2015). https://doi.org/10.1002/eqe. 2548

17. Luco, J.E., De Barros, F.C.P.: Optimal damping between two adjacent elastic structures. Earthq. Eng. Struct. Dyn. 27(7), 649-659 (1998)

18. Gattulli, V., Potenza, F., Lepidi, M.: Damping performance of two simple oscillators coupled by a viscoelastic connection. J. Sound Vib. 332(26), 6934-6948 (2013). https://doi.org/10.1016/j.jsv.2013.08. 037

19. Tubaldi, E., Barbato, M., Dall'Asta, A.: Performance-based seismic risk assessment for buildings equipped with linear and nonlinear viscous dampers. Eng. Struct. 78, 90-99 (2014). https://doi.org/ 10.1016/j.engstruct.2014.04.052

20. Greco, R., Marano, G.C.: Optimum design of viscous dissipative links in wall-frame systems. Struct. Des. Tall Spec. Build. 25(9), 412-428 (2016). https://doi.org/10.1002/tal.1265

21. Gattulli, V., Potenza, F., Spencer, B.F.: Design criteria for dissipative devices in coupled oscillators under seismic excitation. Struct. Control Health Monit. 25(7), e2167 (2018). https://doi.org/10.1002/ stc. 2167

22. Tubaldi, E., Gioiella, L., Scozzese, F., Ragni, L., Asta, A.D.: A design method for viscous dampers connecting adjacent structures. Front. Built Environ. 6(March), 1-14 (2020). https://doi.org/10.3389/ fbuil.2020.00025 
23. Aida, T., Aso, T., Takeshita, K., Takiuchi, T., Fujii, T.: Improvement of the structure damping performance by interconnection. J. Sound Vib. 242(2), 333-353 (2001)

24. Clough, R., Penzien, J.: Dynamics of Structures. McGraw-Hill, New York (1977)

25. Bhattacharjya, S., Chakraborty, S.: Robust optimization of structures subjected to stochastic earthquake with limited information on system parameter uncertainty. Eng. Optim. 43(12), 1311-1330 (2011). https://doi.org/10.1080/0305215X.2011.554545

26. Chakraborty, S., Debbarma, R.: Stochastic earthquake response control of structures by liquid column vibration absorber with uncertain bounded system parameters. Struct. Saf. 33(2), 136-144 (2011). https://doi.org/10.1016/j.strusafe.2011.01.001

27. Roy, B.K., Chakraborty, S., Mihsra, S.K.: Robust optimum design of base isolation system in seismic vibration control of structures under uncertain bounded system parameters. J. Vib. Control 20(5), 786-800 (2014). https://doi.org/10.1177/1077546312466577

28. Xu, J., Spencer, B.F., Lu, X., Chen, X., Lu, L.: Optimization of structures subject to stochastic dynamic loading. Comput. Aided Civil Infrastruct. Eng. 32(8), 657-673 (2017). https://doi.org/10.1111/mice. 12274

29. Schuëller, G.I., Jensen, H.A.: Computational methods in optimization considering uncertainties-an overview. Comput. Methods Appl. Mech. Eng. 198(1), 2-13 (2008). https://doi.org/10.1016/j.cma. 2008.05.004

30. Srinivas, N., Deb, K.: Multiobjective optimization using nondominated sorting in genetic algorithms. Evolut. Comput. 2(3), 221-248 (1994). https://doi.org/10.1162/evco.1994.2.3.221

31. Deb, K.: Multi-Objective Optimization Using Evolutionary Algorithms. Wiley, Chicester (2001)

32. Deb, K., Pratap, A., Agarwal, S., Meyarivan, T.: A fast and elitist multiobjective genetic algorithm: NSGA-II. IEEE Trans. Evolut. Comput. 6(2), 182-197 (2002). https://doi.org/10.1109/4235.996017

33. The MathWorks Inc.: MATLAB Version R2019a Documentation (2019)

34. Federal Emergency Management Agency (FEMA): FEMA P-58-6, Guidelines for Performance-Based Seismic Design of Buildings. Washington, D.C., USA (2018)

35. Jennings, P., Housner, G., Tsai, N.: Simulated earthquake motions for design purposes. In: Proceedings of the 4th World Conference on Earthquake Engineering, vol. 50, pp. 145-160. Santiago, Chile (1969)

36. Marano, G.C., Sgobba, S.: Stochastic energy analysis of seismic isolated bridges. Soil Dyn. Earthq. Eng. 27(8), 759-773 (2007). https://doi.org/10.1016/j.soildyn.2006.12.001

37. Greco, R., Marano, G.C.: Site based stochastic seismic spectra. Soil Dyn. Earthq. Eng. 55, 288-295 (2013). https://doi.org/10.1016/j.soildyn.2013.09.020

38. Greco, R., Fiore, A., Marano, G.C.: The role of modulation function in nonstationary stochastic earthquake model. J. Earthq. Tsunami (2014). https://doi.org/10.1016/j.biomaterials.2007.04.003

39. Acciani, G., Di Modugno, F., Abrescia, A., Marano, G.C.: Integration algorithm for covariance nonstationary dynamic analysis using equivalent stochastic linearization. Math. Comput. Simul. 125, 70-82 (2016). https://doi.org/10.1016/j.matcom.2015.11.006

40. Lin, Y.: Probabilistic Theory of Structural Dynamics. McGraw-Hill, New York (1967)

41. Lutes, L.D., Sarkani, S.: Random Vibrations: Analysis of Structural and Mechanical Systems. Elsevier Butterworth-Heinemann, Oxford (2004)

42. Pareto, V.: Cours d' économique politique. Rouge, Lausanne (1896)

43. Coello, C.A., Christiansen, A.D.: Multiobjective optimization of trusses using genetic algorithms. Comput. Struct. 75(6), 647-660 (2000). https://doi.org/10.1016/S0045-7949(99)00110-8

44. Papadrakakis, M., Lagaros, N.D., Plevris, V.: Multi-objective optimization of skeletal structures under static and seismic loading conditions. Eng. Optim. 34(6), 645-669 (2002). https://doi.org/10.1080/ 03052150215716

45. Lagaros, N.D., Papadrakakis, M., Kokossalakis, G.: Structural optimization using evolutionary algorithms. Comput. Struct. 80(7-8), 571-589 (2002). https://doi.org/10.1016/S0045-7949(02)00027-5

Publisher's Note Springer Nature remains neutral with regard to jurisdictional claims in published maps and institutional affiliations. 